\title{
Artigo/Article
}

\section{Biologia comparativa e comportamento alimentar de Rhodnius neglectus e Rhodnius robustus (Triatominae) sob condições de laboratório}

\author{
Comparative biology and feeding behavior of Rhodnius neglectus and Rhodnius robustus \\ (Triatominae) under laboratory conditions
}

\section{Daniella Barreto-Santana ${ }^{1}$, Jacqueline Starling ${ }^{1}$, Rodrigo Gurgel-Gonçalves ${ }^{1}$ e César Augusto Cuba Cuba ${ }^{1}$}

\section{RESUMO}

Introdução: A competência vetorial de triatomíneos é determinada a partir de estudos sobre biologia e comportamento alimentar em condições de campo e/ou laboratório. Fatores como número de picadas, quantidade de sangue ingerido e tempo de defecação têm implicações na transmissão de tripanosomatídeos. Parâmetros biológicos e comportamentais de Rhodnius neglectus e $R$. robustus foram comparados experimentalmente para estimar diferenças no potencial de transmissão de tripanosomatídeos. Métodos: Os triatomíneos foram observados diariamente para determinar o período de desenvolvimento ninfal, mortalidade, detecção da fonte alimentar, número de picadas, tempo do repasto sanguíneo, quantidade de sangue ingerido, tempo entre o final do repasto e a primeira defecação e frequência de defecação. Resultados: Apesar do período ninfal de $R$. neglectus $(156,4 \pm 25,05 \mathrm{~d})$ ter sido menor que o de $R$. robustus $(204,7 \pm 13,22 \mathrm{~d})$, a mortalidade foi similar entre as espécies $(63,8 \mathrm{e}$ $65 \%$, respectivamente). $R$. robustus e $R$. neglectus detectaram rapidamente a fonte alimentar, especialmente no primeiro estádio (2,5 e 1,6min, respectivamente). Apesar do tempo de repasto sanguíneo ter sido similar entre as espécies, $R$. robustus ingeriu em média uma maior quantidade de sangue em todos os estádios, com maiores valores para as ninfas $\mathrm{V}$. As ninfas de $R$. neglectus picaram mais vezes as fontes alimentares, defecaram mais rápido e mais frequentemente que as de $R$. robustus. Conclusões: Sob as condições de laboratório usadas, $R$. neglectus possui um maior potencial para transmissão de Trypanosoma cruzi e T. rangeli que $R$. robustus, atributo que deve ser avaliado em infecções experimentais.

Palavras-chaves: Triatominae. Rhodnius. Biologia. Competência vetorial. Tripanosomatídeos.

\begin{abstract}
Introduction: The vector competence of triatomine insects is determined by studying their biology and feeding behavior under field and/or laboratory conditions. Factors including the number of bites, the amount of blood ingested and defecation time have implications for trypanosome transmission. The biological and behavioral parameters of Rhodnius neglectus and $R$. robustus were compared under experimental conditions to estimate differences in the potential transmission of trypanosomes. Methods: The insects were observed daily to determine the period of nymphal development, mortality, detection of food source, number of bites, time of blood meal intake, amount of blood ingested, time elapsed between the end of the meal and the first defecation and the frequency of defecation. Results: Although the nymphal development of $R$. neglectus $(156.4 \pm 25.05 \mathrm{~d}$ ) was lower than that of $R$. robustus ( $204.7 \pm 13.22 \mathrm{~d}$ ), the mortality between species was similar (63.8 and $65 \%$ respectively). $R$. robustus and $R$. neglectus quickly located the food source, especially in the first instar ( 2.5 and $1.6 \mathrm{~min}$, respectively). Although the time of blood meal intake was similar between the species, $R$. robustus ingested a larger amount of blood on average at all stages and exhibited higher values for the fifth instar. Nymphs of R. neglectus bit more frequently, and they defecated faster and more often than those of $R$. robustus. Conclusions: Under laboratory conditions, $R$. neglectus has a greater potential for transmission of Trypanosoma cruzi and T. rangeli than does $R$. robustus, an attribute that should be further evaluated in experimental infections.
\end{abstract}

Keywords: Triatominae. Rhodnius. Biology. Vectorial competence. Trypanosomatids.

1. Laboratório de Parasitologia Médica e Biologia de Vetores, Faculdade de Medicina, Universidade de Brasília, Brasília, DF.

Endereço para correspondência: Dra Daniella Barreto-Santana. Lab. Parasitologia Médica e Biologia de Vetores/FM/UnB. Campus Universitário Darcy Ribeiro, Asa Norte, 70910-900 Brasília, DF.

Tel: $51613107-1786$

e-mail: daniellabaag@hotmail.com

Recebido para publicação em 02/09/2010

Aceito em 28/04/2011

\section{INTRODUÇÃO}

Rhodnius neglectus Lent, 1954 e Rhodnius robustus Larrousse, 1927 são espécies de triatomíneos predominantemente silvestres, habitando diferentes espécies de palmeiras no Brasil ${ }^{1-5}$. R. neglectus é uma espécie característica do cerrado no Brasil central com um papel importante na transmissão enzoótica de Trypanosoma cruzi e T. rangeli ${ }^{6}$. Além da invasão de adultos nos domicílios ${ }^{7}$, existem evidências de formação de colônias domiciliares de $R$. neglectus nos Estados de Minas Gerais, São Paulo e Goiás ${ }^{8-10}$, sendo considerado como um vetor secundário na transmissão da doença de Chagas. $R$. robustus (s.l., ver Abad-Franch cols ${ }^{4}$ ) tem ampla distribuição na Amazônia, ocorrendo frequentemente em palmeiras (silvestres e periurbanas) em altas densidades e com relevantes taxas de infecção por tripanosomatídeos ${ }^{1,3,11,12}$. Apesar de não existirem evidências de colonização de $R$. robustus em ambiente domiciliar no Brasil, espécimes adultos infectados por T. cruzi têm invadido casas na região amazônica, sendo potenciais vetores extradomiciliares ou ainda podendo contaminar equipamentos de processamento de alimentos, representando risco de transmissão oral da doença de Chagas ${ }^{13,14}$.

Devido à invasão dessas espécies de Rhodnius ao domicílio, o estudo de parâmetros biológicos que influenciam a capacidade de transmissão de tripanosomatídeos pode contribuir para melhorar a compreensão da importância epidemiológica desses vetores, orientando ações de vigilância das tripanossomíases. Além da suscetibilidade aos parasitos, outras características dos triatomíneos como o comportamento alimentar (detecção da fonte alimentar, número de picadas, tempo de repasto, quantidade de sangue ingerido), e a frequência de dejeções também podem determinar a eficiência na transmissão do T. cruzi ${ }^{15-28}$. Em se tratando de T. rangeli, cujo mecanismo de transmissão é do tipo inoculativo, o número de picadas e o número de interrupções realizadas durante o processo de alimentação também devem ser determinantes da competência vetorial. Parâmetros como duração 
do ciclo de vida e mortalidade também podem ser considerados, pois são variáveis diretamente relacionadas com o tamanho das populações dos vetores. No presente trabalho, parâmetros biológicos e comportamentais de $R$. neglectus e $R$. robustus foram comparados sob condições de laboratório para estimar diferenças no potencial de transmissão de tripanosomatídeos.

\section{MÉTODOS}

\section{Material biológico}

Foram utilizados insetos provenientes das colônias mantidas no Laboratório de Parasitologia Médica e Biologia de Vetores (Faculdade de Medicina, UnB). Os Rhodnius neglectus foram coletados na Reserva Ecológica do IBGE, Brasília, $D^{29}$ e os R. robustus em Benfica, Marabá, $\mathrm{PA}^{30}$.

\section{Desenvolvimento de ovos e estádios ninfais}

Inicialmente foram obtidas aleatoriamente 30 ninfas de quinto estádio das colônias da quarta geração de $R$. neglectus e $R$. robustus, para obtenção de adultos. Após a muda, foram formados três grupos compostos de quatro fêmeas e três machos, para cada espécie.

Os casais de cada espécie foram mantidos em conjunto para a obtenção de ovos em recipientes de plástico $(9,5 \mathrm{~cm}$ de altura x $5,5 \mathrm{~cm}$ de diâmetro), com tampas com uma abertura de $4,5 \mathrm{~cm}$ de diâmetro forradas com uma malha de arame. Estes tinham o fundo forrado com papel filtro e contendo tiras do mesmo papel, dobradas em sanfona ${ }^{16}$.

O número de ovos produzidos está diretamente relacionado à quantidade de sangue ingerido ${ }^{31}$. Por isso, na fase inicial, os insetos adultos dos seis grupos compostos formados foram alimentados duas vezes por semana, em camundongos albinos (Swiss 44, pesando aproximadamente 30 a 35g), provenientes do Biotério Central da Universidade de Brasília, anestesiados com ketamina 80mg/kg + xilazina $10 \mathrm{mg} / \mathrm{kg}$ (via intraperitoneal).

Após a postura, 80 ovos de cada espécie foram agrupados pela data de oviposição para determinar o período de eclosão (embrionamento).Após a eclosão, as ninfas de primeiro estádio foram separadas individualmente em outros recipientes para as posteriores ecdises. Os insetos foram mantidos sob condições controladas de temperatura $\left(28^{\circ} \mathrm{C} \pm 2^{\circ} \mathrm{C}\right)$ e umidade relativa $(75 \% \pm 10 \%)$.

Os insetos foram observados diariamente a fim de determinar os percentuais de mortalidade, o período de embrionamento dos ovos e o período total de desenvolvimento ninfal das espécies em condições de laboratório.

\section{Comportamento alimentar}

Uma arena experimental foi preparada em uma cuba de vidro transparente de $30 \mathrm{~cm}$ de diâmetro. No interior de cada arena foi colocado um camundongo albino imobilizado em uma malha de arame e um espécime de Rhodnius, para o registro dos padrões de alimentação e defecação. A alimentação foi oferecida, aproximadamente, sete dias após cada ecdise e o tempo de oferecimento da fonte alimentar foi de no máximo $30 \mathrm{~min}$. A observação foi contínua durante o processo de alimentação e foi realizada a uma temperatura média de $25^{\circ} \mathrm{C}$. Somente aqueles espécimes que se recusavam a sugar ou sugavam pouca quantidade de sangue eram submetidos à nova tentativa de alimentação. Para ambas as espécies foram estudadas as seguintes variáveis: A) tempo de aproximação: tempo (em minutos) desde que o inseto foi colocado na arena até o momento de introdução da probóscide na pele do camundongo; B) número de picadas: número de vezes que o inseto encostou e introduziu a probóscide na tentativa de encontrar vênulas/arteríolas na pele do camundongo, antes do início evidente do seu repasto; C) número de interrupções do repasto: contabilizou-se o número de interrupções espontâneas do inseto durante o repasto; D) tempo do repasto: tempo (em minutos) entre a primeira picada e o fim da ingestão de sangue; E) quantidade de sangue ingerido: todos os insetos foram pesados antes e imediatamente após o repasto em balança analítica AND HR-200, graduada em mg para determinar a quantidade de sangue ingerido; F) tempo entre o final do repasto e a primeira defecação (em minutos); G) frequência de defecação: proporção de indivíduos que defecaram durante e até 10min após o repasto.

\section{Análises estatísticas}

Foi utilizado inicialmente o teste de Kolmogorov-Smirnov, para verificar a normalidade das variáveis. Para a comparação das médias das variáveis de cada estádio de desenvolvimento, entre as duas espécies, foi utilizado o teste T de Student ou teste de Mann-Whitney. Para comparação das taxas de mortalidade entre as duas espécies foi utilizado o teste qui-quadrado. Para analisar a variação dos parâmetros entre os estádios de desenvolvimento de uma mesma espécie, foram aplicadas análises de variância (ANOVA ou Kruskal-Wallis). Os testes foram realizados no programa Statistica ${ }^{\circledR}$.

\section{Considerações éticas}

O manejo e os cuidados com os animais seguiram os princípios éticos na experimentação animal sugeridos pelo Comitê de Ética no Uso Animal (CEUA) da Universidade de Brasília, Instituto de Ciências Biológicas.

\section{RESULTADOS}

\section{Desenvolvimento de ovos e estádios ninfais}

Dos 80 espécimes de $R$. neglectus, 29 (36,2\%) atingiram a fase adulta. Da ninfa I à ninfa III, os insetos necessitaram, em média, de menos de um mês para alcançarem o estádio seguinte. Dos 80 espécimes de $R$. robustus, 28 (35\%) chegaram à fase adulta. Se comparado com $R$. neglectus, o período de desenvolvimento de $R$. robustus foi significativamente maior em todos os estádios $(\mathrm{p}<0,01)$, exceto para ninfas V (Tabela $\mathbf{1}$ ).

Os percentuais de mortalidade de R. neglectus variaram em relação aos estádios de desenvolvimento. Os maiores foram registrados para as ninfas I e II. As ninfas IV e V apresentaram um percentual similar. Apesar dos percentuais de mortalidade de R. robustus terem sido maiores nas ninfas I e V (Tabela 1 ) não foi detectada diferença significativa entre a taxa de mortalidade do período ninfal de $R$. neglectus e $R$. robustus $\left(\chi^{2}=0,02 ; \mathrm{p}=0,90\right)$.

\section{Tempo de aproximação dos insetos à fonte alimentar}

Rhodnius robustus e $R$. neglectus detectaram rapidamente a fonte alimentar (Figura 1), principalmente no primeiro estádio (em média 2,5 e 1,6min, respectivamente). Não foram detectadas diferenças significativas comparando o valor médio deste parâmetro entre os estádios de ambas as espécies. Entretanto, ao comparar o tempo de aproximação entre os estádios de uma mesma espécie, foram observadas diferenças estatisticamente significativas para $R$. robustus $\left(\mathrm{H}_{5,236}=14,7 ; \mathrm{p}<0,01\right)$ e $R$. neglectus $\left(\mathrm{H}_{5,215}=54,6 ; \mathrm{p}<0,01\right) ; \mathrm{o}$ tempo de aproximação dos insetos à fonte alimentar aumentou de acordo com o desenvolvimento dos estádios ninfais, sendo que na fase adulta o tempo diminuiu em quase $50 \%$ quando comparado com a ninfa V, para ambas as espécies (Figura 1). 
TABELA 1 - Comparação do período de desenvolvimento (em dias) e do percentual de mortalidade de ovos e estádios ninfais de Rhodnius neglectus e Rhodnius robustus.

\begin{tabular}{|c|c|c|c|c|c|c|}
\hline \multirow[b]{3}{*}{ Estádio } & \multicolumn{6}{|c|}{ Duração $(X \pm S$ dias $)$} \\
\hline & \multicolumn{2}{|c|}{ Número de insetos } & \multicolumn{2}{|c|}{ (min - máx) } & \multicolumn{2}{|c|}{ Mortalidade (\%) } \\
\hline & $\begin{array}{l}\text { Rhodnius } \\
\text { neglectus }\end{array}$ & $\begin{array}{l}\text { Rhodnius } \\
\text { robustus }\end{array}$ & $\begin{array}{l}\text { Rhodnius } \\
\text { neglectus }\end{array}$ & $\begin{array}{c}\text { Rhodnius } \\
\text { robustus }\end{array}$ & $\begin{array}{l}\text { Rhodnius } \\
\text { neglectus }\end{array}$ & $\begin{array}{l}\text { Rhodnius } \\
\text { robustus }\end{array}$ \\
\hline Ovo - NI & 80 & 80 & $\begin{array}{c}12,9 \pm 1,70 \\
(7-15)\end{array}$ & $\begin{array}{c}17,5 \pm 1,57 \\
(14-23)\end{array}$ & - $^{*}$ & - $^{*}$ \\
\hline NI - NII & 54 & 53 & $\begin{array}{c}18,6 \pm 6,75 \\
(11-38)\end{array}$ & $\begin{array}{c}23,4 \pm 4,92 \\
(18-48)\end{array}$ & 32,5 & 33,8 \\
\hline NII - NIII & 40 & 49 & $\begin{array}{c}22,5 \pm 5,90 \\
(14-41)\end{array}$ & $\begin{array}{c}28,5 \pm 4,83 \\
(23-45)\end{array}$ & 25,9 & 7,5 \\
\hline NIII - NIV & 33 & 42 & $\begin{array}{c}24,4 \pm 9,11 \\
(16-47)\end{array}$ & $\begin{array}{c}40,7 \pm 5,85 \\
(32-53)\end{array}$ & 17,5 & 14,3 \\
\hline NIV - NV & 31 & 38 & $\begin{array}{c}30,8 \pm 8,59 \\
(18-47)\end{array}$ & $\begin{array}{c}46,3 \pm 11,06 \\
(30-63)\end{array}$ & 6,1 & 9,5 \\
\hline $\mathrm{NV}-\mathrm{AD}$ & 29 & 28 & $\begin{array}{c}45,4 \pm 18,24 \\
(22-88)\end{array}$ & $\begin{array}{c}47,2 \pm 8,92 \\
(32-59)\end{array}$ & 6,5 & 26,3 \\
\hline Período ninfal & - & - & $\begin{array}{c}156,4 \pm 25,05 \\
(86-199)\end{array}$ & $\begin{array}{c}204,7 \pm 13,22 \\
(182-228)\end{array}$ & 63,8 & 65,0 \\
\hline
\end{tabular}

X: média, S: desvio padrão, NI-NV: primeiro a quinto estádio ninfal, AD: adultos. ${ }^{*}$ Os ovos não embrionados foram descartados e substituídos.

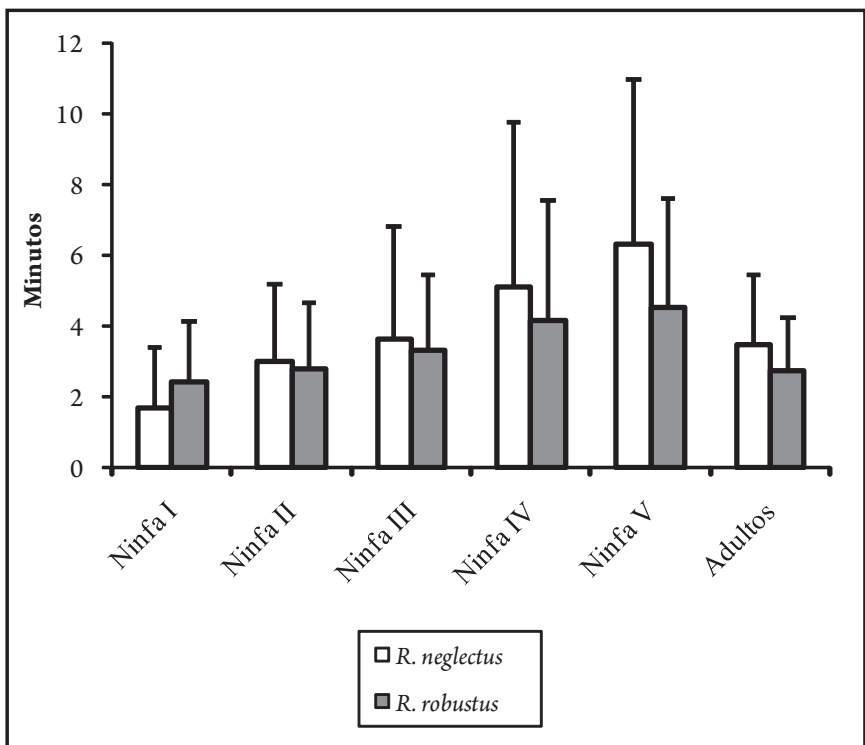

FIGURA 1 - Valor médio do tempo de aproximação dos insetos a fonte alimentar para os estádios de desenvolvimento de Rhodnius neglectus e Rhodnius robustus. As barras representam o desvio padrão da média.

\section{Número de picadas}

Não foram observadas diferenças estatisticamente significativas comparando o número médio de picadas das ninfas I, II, III e adultos de R. neglectus e R. robustus. Entretanto as ninfas IV e V de R. neglectus apresentaram um número médio de picadas significativamente maior que as de $R$. robustus (Figura 2).

O número de picadas foi diferente entre os estádios de $R$. robustus $\left(\mathrm{H}_{5,236}=82,7 ; \mathrm{p}<0,01\right)$ e $R$. neglectus $\left(\mathrm{H}_{5,215}=40,6 ; \mathrm{p}<0,01\right)$. Para ambas as espécies, as ninfas I foram as que apresentaram o maior número de picadas, sendo este reduzido durante o desenvolvimento dos estádios ninfais até a fase adulta, que apresentou o menor número de picadas (Figura 2).

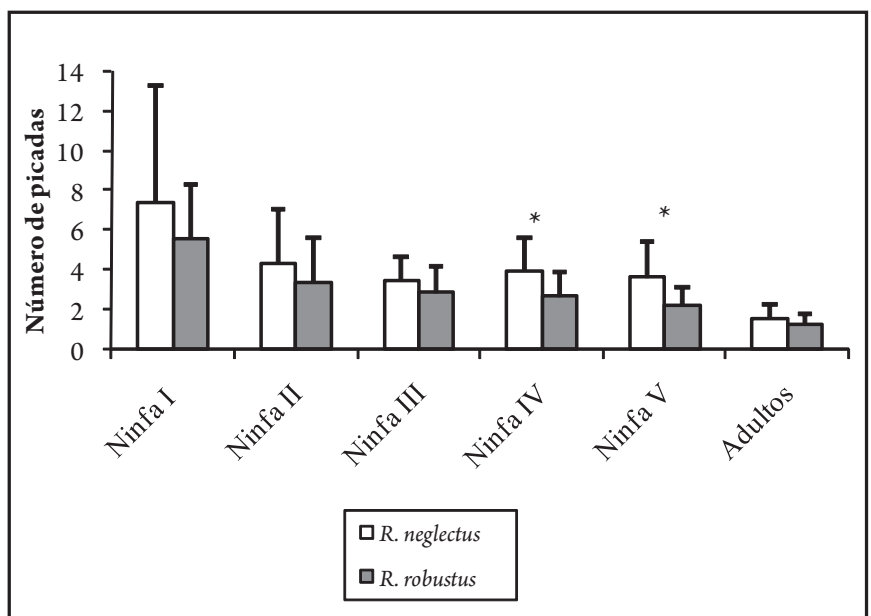

FIGURA 2 - Valor médio do número de picadas de Rhodnius neglectus e Rhodnius robustus realizadas na fonte alimentar.

As barras representam o desvio padrão das médias. ${ }^{*}$ Diferença significativa comparando as médias de $R$. neglectus e $R$. robustus $(\mathrm{p}<0,01)$

\section{Número de interrupções}

Não foram detectadas diferenças estatisticamente significativas do número médio de interrupções dos estádios entre $R$. neglectus $\mathrm{e}$ $R$. robustus nem entre os estádios de desenvolvimento de cada espécie. Considerando todos os insetos observados, o número médio foi de 1,5 interrupções por repasto.

\section{Tempo de repasto e quantidade de sangue ingerido em cada estádio ninfal}

Apesar do tempo de repasto sanguíneo ter sido similar entre as espécies, $R$. robustus ingeriu em média uma maior quantidade de sangue em todos os estádios, com maiores valores para a ninfa $\mathrm{V}$ (Figura 3). O tempo total de repasto variou significativamente entre os estádios de desenvolvimento para $R$. neglectus $\left(\mathrm{F}_{5,208}=5,6 ; \mathrm{p}<0,01\right)$ e $R$. robustus $\left(\mathrm{F}_{5,228}=5,6 ; \mathrm{p}<0,01\right)$, aumentando ao longo do 


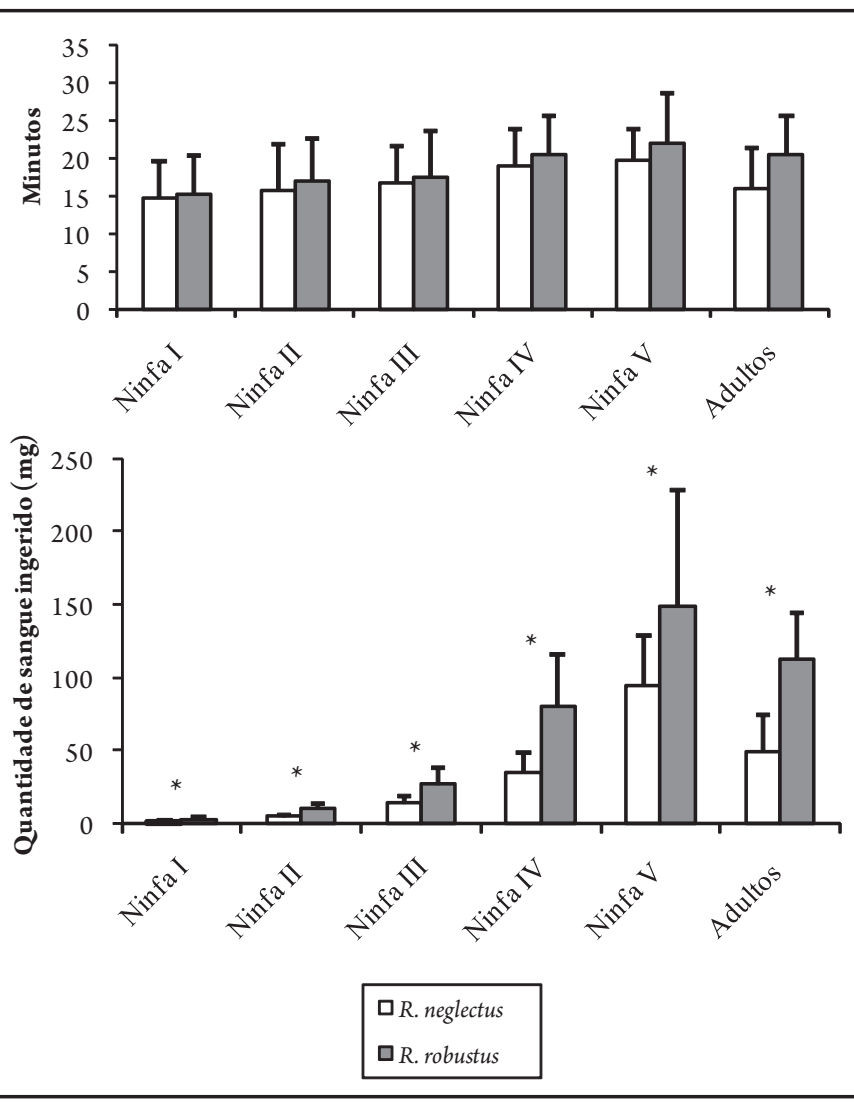

FIGURA 3 - Valor médio do tempo total do repasto sanguíneo (em minutos, acima) e da quantidade de sangue ingerido (em $\mathrm{mg}$, abaixo) para Rhodnius neglectus e Rhodnius robustus.

As barras representam o desvio padrão das médias. ${ }^{*}$ Diferença significativa comparando as médias de $R$. neglectus e R. robustus ( $\mathrm{p}<0,01)$.

desenvolvimento e diminuindo na fase adulta. Também houve diferença significativa na quantidade de sangue ingerido entre os estádios ninfais para $R$. neglectus $\left(\mathrm{H}_{5,213}=188,3 ; \mathrm{p}<0,01\right) \mathrm{e}$ R. robustus $\left(\mathrm{H}_{5,207}=163,7 ; \mathrm{p}<0,01\right)$. Para ambas as espécies, a quantidade de sangue ingerido foi crescente com o decorrer do desenvolvimento dos insetos, alcançando o pico na ninfa V (Figura 3).

\section{Tempo entre o final do repasto e a primeira defecação e frequência de defecação}

Em média, as ninfas de $R$. neglectus defecaram mais rápido e mais frequentemente que as de $R$. robustus, principalmente a ninfa III (Figura 4). Para R. neglectus as ninfas I não defecaram durante o período de 10min estabelecido para avaliar esse experimento. Comparando o tempo entre o final do repasto e a primeira defecação dos outros quatro estádios e adultos de $R$. neglectus, não foi detectada diferença significativa $\left(\mathrm{H}_{4,65}=4,2 ; \mathrm{p}=0,37\right)$. No caso de $R$. robustus, essa diferença foi significativa, a ninfa I demorou mais tempo para defecar em relação aos outros estádios de desenvolvimento $\left(\mathrm{H}_{5,64}=19,4 ; \mathrm{p}<0,01\right)$. Em ambas as espécies o maior percentual de indivíduos que defecaram em até 10 min ocorreu no estádio V (Figura 4).

\section{DISCUSSÃO}

Os resultados do presente trabalho mostram diferenças biológicas e comportamentais entre $R$. neglectus e $R$. robustus que podem influenciar o mecanismo de transmissão de $T$. rangeli e T. cruzi, determinando a competência vetorial dessas espécies de

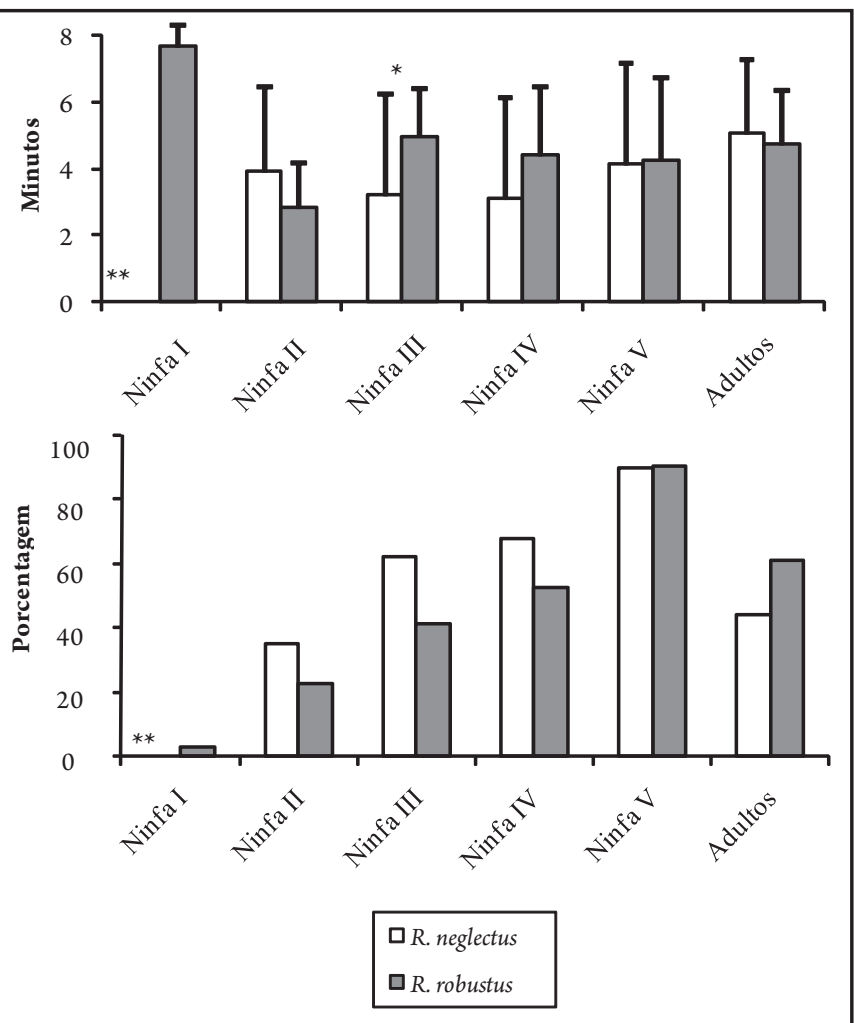

FIGURA 4 - Valor médio do tempo entre o final do repasto até a primeira defecação (em minutos, acima) e frequência de defecações (em porcentagem, abaixo) para Rhodnius neglectus e Rhodnius robustus de acordo com o estádio de desenvolvimento.

As barras representam o desvio padrão das médias. ${ }^{*}$ Diferença significativa comparando as médias de $R$. neglectus e $R$. robustus ( $<<0,01$ ).**Não foi possível comparar pois nenhuma ninfa I de $R$. neglectus defecou antes do tempo estabelecido (10min).

triatomíneos. Apesar do período ninfal de R. neglectus ter sido menor que o de $R$. robustus, a mortalidade foi similar entre as espécies. $R$. robustus e $R$. neglectus detectaram rapidamente a fonte alimentar, principalmente no primeiro estádio. Apesar do tempo de repasto sanguíneo ter sido similar entre as espécies, $R$. robustus ingeriu em média uma maior quantidade de sangue em todos os estádios. As ninfas de $R$. neglectus picaram mais vezes as fontes alimentares, defecaram mais rápido e mais frequentemente que as de $R$. robustus. Em conjunto essas evidências sugerem que, em comparação a $R$. robustus, $R$. neglectus possui um maior potencial para transmissão de $T$. rangeli e $T$. cruzi.

Os períodos de desenvolvimento ninfal encontrados no presente trabalho estão dentro dos limites já descritos para $R$. neglectus (75 a 301 dias) $)^{32-34}$ e R. robustus (72 a 364 dias) $)^{33,35-37}$. Essa grande variação geralmente tem sido associada às condições experimentais de alimentação, temperatura e umidade em que os triatomíneos são submetidos. Porém, pelo menos para $R$. robustus, atualmente considerado como um conjunto de espécies crípticas ${ }^{4}$, as diferenças nos períodos de desenvolvimento ninfal também poderiam ser explicadas por diferenças genéticas e, consequentemente, fisiológicas entre as linhagens de $R$. robustus descritas. Futuros estudos comparando, sob as mesmas condições experimentais, os ciclos de vida dessas diferentes linhagens poderiam esclarecer essa questão e ainda auxiliar a delimitação dessas linhagens a partir de critérios biológicos. Parâmetros biológicos como período de desenvolvimento ninfal e mortalidade influenciam diretamente o tamanho das 
populações; quanto menor o período de desenvolvimento e taxa de mortalidade, maior o tamanho das populações de triatomíneos nos ambientes silvestres e domésticos. A partir dos resultados do presente trabalho seria esperado um maior tamanho populacional para R. neglectus.

O maior percentual de mortalidade foi observado no primeiro estádio para ambas as espécies. Isso pode ter ocorrido pela dificuldade na realização do primeiro repasto devido à fragilidade do aparelho bucal. Problemas durante a última muda podem explicar a mortalidade dos insetos no estádio $V$, o que também foi observado para R. prolixus por Lent \& Valderrama ${ }^{38}$.

Rhodnius robustus e $R$. neglectus detectaram rapidamente a fonte alimentar, geralmente em menos de $5 \mathrm{~min}$. Esse tempo médio de aproximação foi similar ao observado para R. pictipes ${ }^{16}$, Meccus longipennis ${ }^{22}$, Triatoma infestans e $R$. prolixus ${ }^{15}$. De acordo com Guarneri $\operatorname{cols}^{39}$, quanto mais eficiente é a espécie durante o processo de alimentação, mais curto será o seu tempo de contato com o hospedeiro e maior será sua chance de sobrevivência. Dessa forma, ambas as espécies estudadas podem ser consideradas eficientes no processo de alimentação, já que mostraram similar comportamento para detecção da fonte alimentar. $\mathrm{O}$ menor tempo de aproximação à fonte alimentar observado no primeiro estádio evidencia um comportamento alimentar inato de ambas as espécies na detecção de camundongos. Futuros estudos apresentando diferentes espécies de vertebrados como fontes alimentares para as ninfas I, podem esclarecer a influência da fonte alimentar no comportamento de aproximação.

No presente trabalho, $R$. neglectus apresentou um maior número de picadas em todos os estádios, se comparado a $R$. robustus, o que aumentaria a probabilidade de transmissão de $T$. rangeli. Considerando que antes de iniciar a hematofagia os triatomíneos injetam saliva no hospedeiro, quanto maior o número de tentativas de picadas, maior a quantidade inoculada de tripomastigotas metacíclicos de $T$. rangeli, provenientes das glândulas salivares de insetos infectados. Em ambas as espécies observou-se uma diminuição do número de picadas com a aproximação da fase adulta, e isso poderia estar relacionado a uma maior eficiência na detecção do vaso sanguíneo ao longo do desenvolvimento ninfal até a fase adulta. Futuros experimentos comparando a infecção experimental por $T$. rangeli em ambas as espécies poderão confirmar o maior potencial de R. neglectus na transmissão desse parasito. Já o número de interrupções não variou significativamente entre as espécies e entre os estádios. A maioria das interrupções ocorreu pela irritabilidade do hospedeiro, como já observado por Bar cols ${ }^{20}$. No caso de infecções experimentais com $T$. rangeli, as interrupções podem ainda estar relacionadas com a ação patogênica deste parasito, que dificultaria a capacidade hematofágica dos triatomíneos ${ }^{24}$.

Já a duração do repasto e a quantidade de sangue ingerido aumentaram ao longo do desenvolvimento ninfal e diminuíram na fase adulta. Silva ${ }^{40}$ estudando seis espécies de Rhodnius e Arévalo $\operatorname{cols}^{25}$ comparando os padrões de alimentação e defecação de $R$. colombiensis e R. prolixus, também registraram uma maior quantidade de sangue ingerido no estádio $\mathrm{V}$ e o decréscimo na fase adulta. A maior necessidade de sangue ingerido nas ninfas $\mathrm{V}$ deve estar relacionada à aquisição de novas estruturas anatômicas e mudanças fisiológicas durante a passagem para a fase adulta.

De acordo com Zeledón cols ${ }^{15}$, as espécies de triatomíneos que defecam durante os primeiros 5 a 10min após a alimentação podem ser consideradas transmissoras eficazes de T. cruzi. Dessa forma, todas as ninfas observadas no presente trabalho (com exceção das ninfas I de R. neglectus) poderiam ser consideradas eficazes para a transmissão de T. cruzi, pois apresentaram o tempo médio menor ou igual à $5 \mathrm{~min}$. Segundo Nattero $\operatorname{cols}^{41}$, a defecação depende da quantidade de sangue ingerido. Quanto maior a quantidade de sangue ingerido, menor será o tempo entre o final do repasto e a primeira defecação e maior será a possibilidade de infecção do hospedeiro por T. cruzi. Adicionalmente, Sant'Anna cols ${ }^{42}$ mostraram que $R$. robustus apresenta maiores taxas de ingestão de sangue quando comparado a $R$. neglectus. Analisando esse parâmetro, R. robustus seria um vetor mais eficiente que $R$. neglectus na transmissão deste parasito. Entretanto, no presente trabalho, R. neglectus defecou mais rápido (principalmente o estádio III) e mais frequentemente quando comparado ao $R$. robustus, mesmo ingerindo uma quantidade de sangue significativamente menor. Uma maior frequência de defecação no terceiro estádio também foi observada por Aldana cols ${ }^{19}$ comparando estádios de desenvolvimento de quatro espécies de Rhodnius.

Outros fatores devem influenciar os padrões de defecação e a competência dessas espécies de Rhodnius na transmissão de tripanosomatídeos, entre eles a afinidade parasito-vetor e a variabilidade genética das linhagens de parasitos que podem determinar a infecção no vetor ${ }^{24,43}$. Nesse sentido, Martins cols ${ }^{18}$ mostraram que $R$. neglectus era mais suscetível a $T$. cruzi que R. robustus e Carvalho-Moreira cols ${ }^{21}$ chegaram a mesma conclusão comparando $R$. neglectus e T. pseudomaculata. Futuros estudos comparativos de infecção experimental dessas espécies de Rhodnius frente a diferentes linhagens de T. cruzi e T. rangeli poderão esclarecer o papel da biologia do vetor, do parasito e da interação entre ambos na determinação da transmissão desses tripanosomatídeos ao homem.

\section{AGRADECIMENTOS}

Ao estagiário Jonatas C.B. Ferreira pela colaboração na manutenção da colônia de triatomíneos; aos técnicos Shigueru Ofugi e Walcymar P. Santiago, do Laboratório de Doença de Chagas (NMT-UnB) pelo apoio técnico; e aos revisores anônimos pelas críticas e sugestões.

\section{CONFLITO DE INTERESSE}

Os autores declaram não haver nenhum tipo de conflito de interesse no desenvolvimento do estudo.

\section{REFERÊNCIAS}

1. Miles MA, Arias JR, Souza AA. Chagas disease in the Amazon basin: V. Periurban palms as habitats of Rhodnius robustus and Rhodnius pictipes - triatomine vectors of Chagas disease. Mem Inst Oswaldo Cruz 1983; 78: 391-398.

2. Diotaiuti L, Dias JC. Occurrence and biology of Rhodnius neglectus Lent, 1954 in palm trees of suburban areas of Belo Horizonte, Minas Gerais. Mem Inst Oswaldo Cruz 1984; 79: 293-301.

3. Abad-Franch F, Monteiro FA. Biogeography and evolution of Amazonian triatomines (Heteroptera: Reduviidae): implications for Chagas disease surveillance in humid forest ecoregions. Mem Inst Oswaldo Cruz 2007; 102 (supl I): 57-70.

4. Abad-Franch F, Monteiro FA, Jaramillo NO, Gurgel-Gonçalves R, Dias FBS, Diotaiuti L. Ecology, evolution and the long-term surveillance of vector-borne Chagas disease: A multi-scale appraisal of the tribe Rhodniini (Triatominae). Acta Trop 2009; 112:159-177. 
5. Gurgel-Gonçalves R, Cuba CAC. Predicting the potential geographical distribution of Rhodnius neglectus (Hemiptera, Reduviidae) based on ecological niche modeling. J Med Entomol 2009; 46:952-960.

6. Gurgel-Gonçalves R, Ramalho ED, Duarte MA, Palma ART, Abad-Franch F, Carranza JC, et al. Enzootic transmission of Trypanosoma cruzi and Trypanosoma rangeli in Federal District of Brazil. Rev Inst Med Trop São Paulo 2004; 46:323-330.

7. Gurgel-Gonçalves R, Abad-Franch F, Ferreira JBC, Santana DB, Cuba CAC. Is Rhodnius prolixus (Triatominae) invading houses in central Brazil? Acta Trop 2008; 107: 90-98.

8. Barretto MP, Siqueira AF, Ferriolli FF, Carvalheiro JR. Estudos sobre reservatórios e vetores do Trypanosoma cruzi.XXIII. Observações sobre criadouros do Rhodnius neglectus Lent, 1954 em biótopos artificiais (Hemiptera, Reduviidae). Rev Inst Med Trop São Paulo 1968; 10:163-170.

9. Silva RA, Bonifácio PR, Wanderley DMV. Doença de Chagas no estado de São Paulo: comparação entre pesquisa ativa de triatomíneos em domicílios e notificação de sua presença pela população em área sob vigilância entomológica. Rev Soc Bras Med Trop 1999; 32:653-659.

10. Oliveira AWS, Silva IG. Distribuição geográfica e indicadores entomológicos de triatomíneos sinantrópicos capturados no Estado de Goiás. Rev Soc Bras Med Trop 2007; 40:204-208.

11. Carcavallo RU, Martínez-Silva R, Otero MAA, Tonn RJ. Infección natural de Rhodnius robustus Larrousse y Rhodnius pictipes Stal por T. cruzi y T. rangeli en Venezuela. Bol Dir Malariol San Amb 1975; 15:117-120.

12. Feliciangeli MD, Dujardin JP, Bastrenta B, Mazzarri M, Villegas J, Flores M, et al. Is Rhodnius robustus (Hemiptera: Rediviidae) responsible for Chagas disease transmission in Western Venezuela? Trop Med Int Health 2002; 7: 280-287.

13. Coura JR, Junqueira ACV, Fernandes O, Valente SAS, Miles MA. Emerging Chagas Diease in Amazonian Brazil. Trends Parasitol 2002; 18:171-176.

14. Aguilar HM, Abad-Franch F, Dias JCP, Junqueira ACV, Coura JR. Chagas disease in the Amazon Region. Mem Inst Oswaldo Cruz 2007; 102:47-55.

15. Zeledón R, Alvarado R, Jirón JF. Observations on the feeding and defecation patterns of three triatomine species (Hemiptera, Reduviidae). Acta Trop 1977; 34:65-77.

16. Rocha DS, Jurberg J, Galvão C. Biologia do Rhodnius pictipes Stal, 1872 em condições de laboratório (Hemiptera, Reduviidae, Triatominae). Mem Inst Oswaldo Cruz 1994; vol. 89: 265-270.

17. Diotaiuti L, Penido CM, Pires HHR, Dias JCP. Dinâmica de alimentação e dejeção do Triatoma sordida. Rev Soc Bras Med Trop 1995; 28:195-198.

18. Martins LPA, Rosa JA, Castanho REP, Sauniti GL, Medeiros Jr H. Susceptibilidade de Rhodnius neglectus, Rhodnius robustus e Triatoma infestans (Hemiptera, Reduviidae, Triatominae) à infecção por duas cepas de Trypanosoma cruzi (Kinetoplastidae, Trypanosomatidae) utilizando xenodiagnóstico artificial. Rev Soc Bras Med Trop 2000; 33:559-563.

19. Aldana E, Lizano E, Rodriguez M, Valderrama A. Alimentación y defecación en triatominos del género Rhodnius (Hemiptera, Reduviidae) alimentados con sangre humana. Rev Biol Trop 2001; 49:693-696

20. Bar ME, Milano AMF, Damborsky MP, Oscherov EB, Avalos G. Patrones de alimentación y de defecación de Triatoma rubrovaria (Heteroptera: Reduviidae) bajo condiciones de laboratorio. Rev Soc Entomol Argent 2003; 62:107-113.

21. Carvalho-Moreira CJ, Spata MCD, Coura JR, Garcia ES, Azambuja P, Gonzalez MS, et al. In vivo and in vitro metacyclogenesis tests of two strains of Trypanosoma cruzi in the triatomine vectors Triatoma pseudomaculata and Rhodnius neglectus: short/long-term and comparative study. Exp Parasitol 2003; 103:102-111.

22. Martinez-Ibarra JA, Grant-Guillen Y, Martinez-Grant DM. Feeding, defecation, and development times of Meccus longipennis Usinger, 1939 (Hemiptera, Reduviidae, Triatominae) under laboratory conditions. Mem Inst Oswaldo Cruz 2003; 98: 899-903.

23. Rocha DS, Santos CM, Cunha V, Jurberg J, Galvão G. Ciclo biológico em laboratório de Rhodnius brethesi Matta 1919 (Hemiptera, Reduviidae, Triatominae), potencial vetor silvestre da doença de Chagas na Amazônia. Mem Inst Oswaldo Cruz 2004; 99:591-595.

24. Azambuja P, Garcia ES. Trypanosoma rangeli interactions within the vector Rhodnius prolixus: a mini review. Mem Inst Oswaldo Cruz 2005; 100:567-572.
25. Arévalo A, Carranza JC, Guhl F, Clavijo JA, Vallejo GA. Comparación de los patrones de alimentación y defecación de Rhodnius colombiensis y Rhodnius prolixus (Hemiptera, Reduviidae, Triatominae) en condiciones de laboratório. Biomedica 2007; 27 (supl.1):101-109.

26. Rodríguez CS, Carrizo AS, Crocco LB. Comparison of feeding and defecation patterns between fifth-instar nymphs of Triatoma patagonica (Del Ponte, 1929) and Triatoma infestans (Klug, 1934) under laboratory conditions. Rev Soc Bras Med Trop 2008; 41:330-333.

27. Oliveira TG, Carvalho-Costa FA, Sarquis O, Lima MM. Feeding and defecation patterns of Rhodnius nasutus (Hemiptera, Reduviidae), a triatomine native to an area endemic for Chagas disease in the state of Ceará, Brazil. Am J Trop Med Hyg 2009; 81:651-655.

28. Pérez AGR, Moreno JE, Colina L. Estudio comparativo de la susceptibilidad de cuatro especies del genero Rhodnius a la infección experimental con Trypanosoma cruzi. Bol Mal Salud Amb 2009; 49:135-142.

29. Gurgel-Gonçalves R, Duarte MA, Ramalho ED, Palma ART, Romana CA, CubaCuba CA. Distribuição espacial de populações de triatomíneos (Hemiptera: Reduviidae) em palmeiras da espécie Mauritia flexuosa no Distrito Federal, Brasil Rev Soc Bras Med Trop 2004; 37: 241-247.

30. Mejía GD. Aspectos ecológicos de populações silvestres de Triatomina (Hemiptera: Reduviidae) em palmeiras Attalea speciosa (Arecaceae) e estimativa do risco ambiental para a transmissão de Trypanosomatidae (Kinetoplastida) em uma frente pioneira de desmatamento (Pará-Brasil). [Dissertação de Mestrado]. [Brasília]: Universidade de Brasília; 2005. 124p.

31. Friend WG, Choy CTH, Cartwright E. The effect of nutrient intake on the development and the egg prodution of Rhodnius prolixus, Stal (Hemiptera: Reduviidae). Can J Zool 1965; 43: 891-904.

32. Diotaiuti L, Dias JC. Estudo comparativo do ciclo evolutivo de Rhodniu neglectus alimentados em pombos e camundongos. Rev Soc Bras Med Trop $1987 ; 20: 95-100$

33. Canale MD, Jurberg J, Carcavallo RU, Galvão C, Galíndez Giron I, Mena Segura CA, et al. Bionomics of some species. In: Carcavallo RU, Galíndez Girón I, Jurberg J, Lent H, editores. Atlas of Chagas Disease Vectors in the America. Vol. III. Rio de Janeiro: Editora FIOCRUZ; 1999. p. 839-890.

34. Rocha DS, Jurberg J, Carcavallo RU. Influence of the temperature and humidity on the biology of Rhodnius neglectus Lent, 1954 in laboratory conditions. Rev Soc Bras Med Trop 2001; 34:357-363.

35. Tonn R, Carcavallo R, Ortega R. Notas sobre la biologia, ecologia y distribución geográfica de Rhodnius robustus Larousse, 1927 (Hemiptera: Reduviidae) Bol Dir Malariol San Amb 1976; 26:158-171.

36. Jurberg J, Rangel EF. Observações sobre Rhodnius robustus Larrousse, 1927 e Rhodnius pallescens Barber, 1932 (Hemiptera, Reduviidae, Triatominae) Rev Bras Biol 1980; 40:569-577.

37. Rocha DS, Jurberg J, Carcavallo RU. Influence of temperature and humidity on the nymphal development of Rhodnius robustus. Rev Saude Publica $2001 ; 35: 400-406$

38. Lent H, Valderrama A. Observações, em laboratório, sobre o ciclo evolutivo de Rhodnius prolixus Stal, 1959, R. pictipes Stal, 1872 e R. neivai Lent, 1953. Rev Bras Biol 1977; 37:325-344.

39. Guarneri AA, Carvalho MG, Pereira MH, Diotaiuti L. Potencial biológico do Triatoma brasiliensis. Cad Saude Publica 2000; 16 (supl. 2):101-104.

40. Silva IG. Influência da temperatura na biologia de 18 espécies de triatomíneos (Hemiptera, Reduviidae) e no xenodiagnóstico [Dissertação de Mestrado]. [Curitiba]: Universidade Federal do Paraná; 1985. 169p.

41. Nattero J, Crocco LB, Rodriguez CS. Feeding and defaecation behaviour of Triatoma patagonica (Del Ponte, 1929) (Hemiptera: Reduviidae). Mem Inst Oswaldo Cruz 2002; 97: 1063-1065.

42. Sant'Anna MRV, Diotaiuti L, Gontijo AF, Gontijo NF, Pereira MH. Feeding behaviour of morphologically similar Rhodnius species: inûuence of mechanical characteristics and salivary function. J Insect Physiol 2001; 47:1459-1465.

43. Vallejo GA, Guhl F, Schaub. Triatominae-Trypanosoma cruzi/T. rangeli: Vectorparasite interactions. Acta Trop 2009; 110:137-147. 